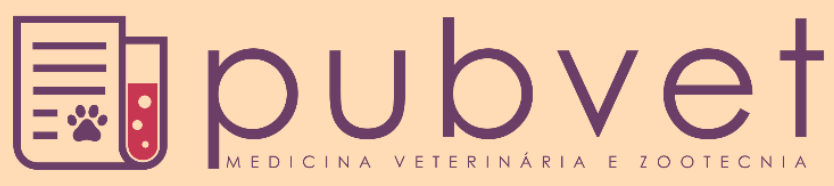

https://doi.org/10.31533/pubvet.v15n11a973.1-7

\title{
Estafilectomia e rinoplastia em Bulldog Francês
}

\author{
Débora Rodrigues Ferreira $^{\bullet}$, Ivan Torres Gregório da $\operatorname{Silva}^{2}{ }^{\ominus}$, Flavia Alves Gomes ${ }^{\circ}$, Thainá \\ Elis Fonseca de Aquino ${ }^{3}$
}

'Discente em Medicina Veterinária pela Universidade Federal Fluminense, Rio de Janeiro, Brasil.

${ }^{2}$ Pós-graduado em Cirurgia de Tecidos Moles pela Anclivepa-SP, Rio de Janeiro, Brasil.

${ }^{3}$ Discente de Medicina Veterinária pelo Centro Universitário Univeritas, São Gonçalo, Brasil.

*Autor para correspondência, E-mail: deborarf@id.uff.br

\begin{abstract}
Resumo. A síndrome braquicefálica é um conjunto de alterações anatômicas e fisiológicas, que dificultam a passagem de ar nas vias aéreas. Essa síndrome combina a estenose de narinas e alongamento do palato mole como alterações primárias, que levam a alterações secundárias. Em função desses fatores, o animal faz maior esforço respiratório, apresentando sinais clínicos como ronco durante o sono e acordado, intolerância ao exercício físico, disfagia, espirro reverso e cianose. O relato objetiva apresentar as técnicas cirúrgicas estafilectomia e rinoplastia utilizadas para correção da angústia respiratória em canino da raça Bulldog Francês.
\end{abstract}

Palavras-chave: Braquicefálico, cirúrgica, estenose, palato mole

\section{French Bulldog staphylectomy and rhinoplasty}

Abstract. The brachycephalic syndrome is a set of anatomical and physiological changes that hinder the passage of air through the airways. This syndrome combines nostril stenosis and soft palate elongation as primary changes, which lead to secondary changes. Because of these factors, the animal makes greater respiratory effort, presenting clinical signs such as snoring during sleep and awake, intolerance to physical exercise, dysphagia, reverse sneezing and cyanosis. The report aims to present the surgical techniques used for staphylectomy and rhinoplasty to correct respiratory distress in a French Bulldog canine.

Key words: Brachycephalic, surgical, stenosis, soft palate

\section{Introdução}

A síndrome braquicefálica é a patologia mais comum entre algumas raças caninas como Pug, Boxer, Shih-tzu, Bulldog francês e Bulldog inglês (Slatter, 2007). A combinação de estenose de narina e palato mole alongado configuram uma série de alterações respiratórias causadas pela anatomia diferenciada desses animais, podendo causar alterações secundárias, como a eversão dos sacos laríngeos, colapso de traqueia, eversão das tonsilas, colapso de faringe, colapso de epiglote e macroglossia (Fossum, 2014).

Como outras alterações secundárias, podem ocorrer a dilatação esofágica e gástrica, como resultado do grande esforço respiratório inspiratório, devido a obstrução das narinas dos animais braquicefálicos. Nesses pacientes, é comum ocorrer flatulência, vômitos e regurgitação. Toda essa grande deglutição de ar pode ocasionar a distensão crônica do trato digestivo (Jericó et al., 2015). Além disso, a síndrome é progressiva, e quando não tratada cirurgicamente em seus primeiros indícios, pode acarretar doenças dermatológicas e no trato intestinal, uma vez que, a dificuldade respiratória causa um déficit de oxigênio nas células. (Fossum, 2014). Na maioria dos casos, os tutores não identificam a síndrome das vias áreas em cães braquicefálicos em seus animais, mesmo com apresentação de sintomas (Lopes \& Vasconcelos, 
2020). O tratamento cirúrgico é o único método eficaz, mas algumas condutas podem ser adotadas para melhorar as condições de vida dos animais, como lugares arejados, com temperaturas amenas e evitar exercícios excessivos (Lameu et al., 2020).

Os procedimentos cirúrgicos denominados estafilectomia que consiste na remoção da porção terminal do palato mole (Franco et al., 2015) e a rinoplastia que remodela a estrutura nasal, tanto óssea quanto a parte cartilaginosa garantem ao paciente o retorno das funções mastigatórias e respiratórias, com ótima aparência estética (Reinstein et al., 2020). Esse relato objetiva apresentar as complicações da síndrome, seus meios de diagnóstico e seu tratamento cirúrgico, impactando positivamente na vida do animal.

\section{Relato de caso e discussão}

Animal Adam, da raça Bulldog Francês de três anos de idade e $14 \mathrm{~kg}$. Foi ao atendimento médico após apresentar a seguinte sintomatologia: dificuldade severa ao exercício, disfagia, regurgitação frequente, ronco enquanto acordado e dormindo e espirro reverso.

Foi possível realizar exames físicos e o paciente em questão foi diagnosticado como grau III na escala Brachycephalic obstructive airway syndrome (BOAS), que é um parâmetro americano que descreve as alterações nos cães braquicefálicos e seus níveis, visto que esses animais possuem os ossos craniais mais curtos que os tecidos moles, essa escala combina todos os sinais clínicos recorrentemente observados em animais braquicefálicos. Este paciente já possuía sintomatologia bem avançada e com a qualidade de vida severamente afetada. Com ausculta pulmonar e após os exames clínicos, constatou-se a estenose de narinas e palato mole alongado. Dessa forma, foram solicitados exames cardiológicos, hematológicos e radiográficos.

O animal foi constatado como saudável tanto no exame radiológico (Imagem 1), como nos exames de sangue e cardiológico (Anexos 1 - 4). Devido aos bons resultados nos exames pré-operatórios, o paciente estava apto para correção cirúrgica da síndrome braquicefálica.

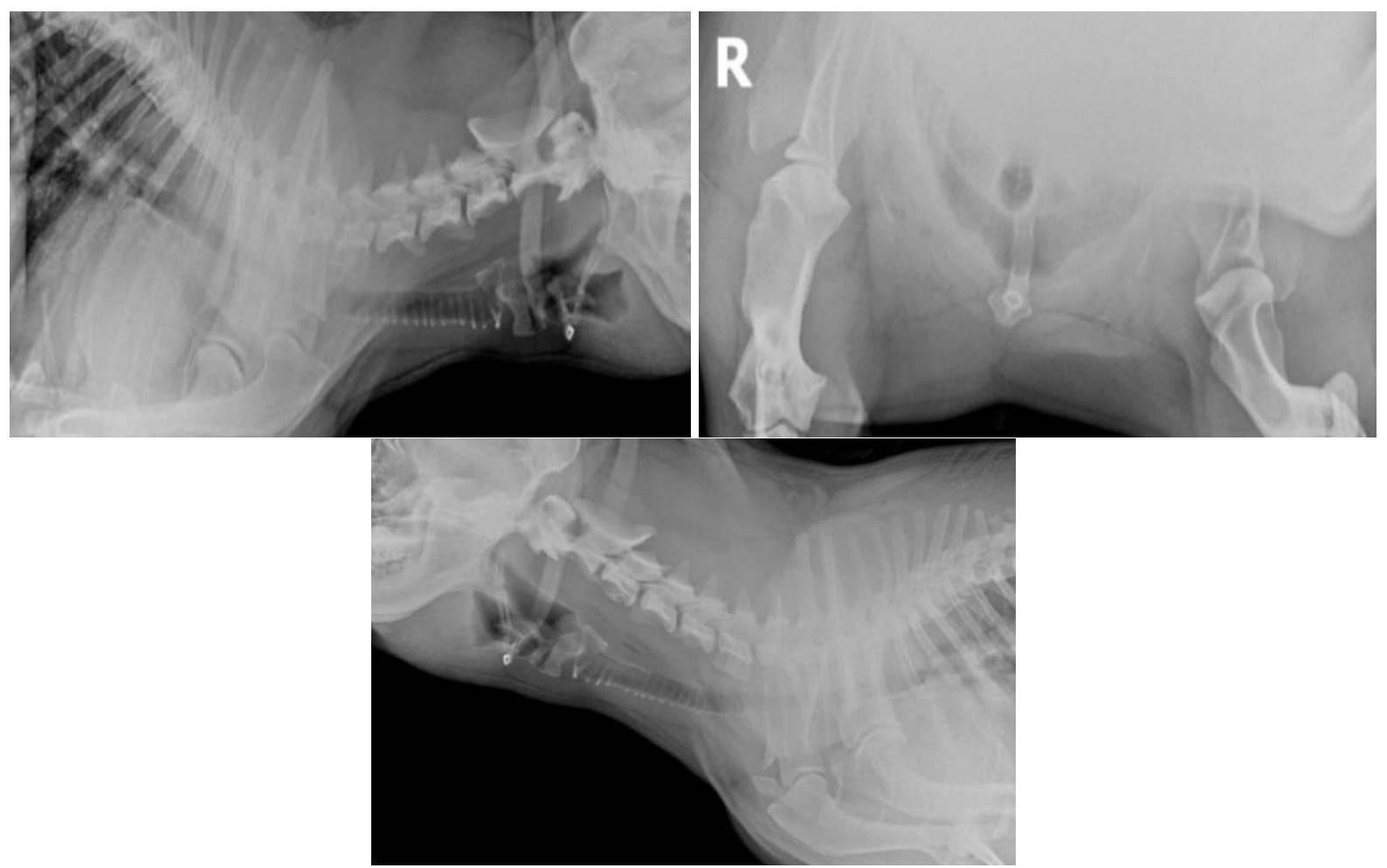

Imagem 1. Laudo do exame radiográfico. Estruturas de faringe e laringe dentro da normalidade radiográfica; traqueia com diâmetro preservado e trajeto anatômico; esôfago sem sinais de alterações no estudo radiográfico; demais estruturas cervicais visualizadas dentro da normalidade radiográfica. 
Foram iniciadas as seguintes medicações prévias; rutina $30 \mathrm{mg} / \mathrm{kg} 8 / 8 \mathrm{~h}$ e Prednisolona $1 \mathrm{mg} / \mathrm{kg}$ 24/24h, três dias antes da cirurgia.

No dia da cirurgia, o paciente foi pesado atestando $14 \mathrm{~kg}$. Assim, foram feitos os cálculos para as medicações anestésicas. Como medicação pré-anestésica, o animal recebeu 0,3 $\mathrm{mg} / \mathrm{kg}$ de Metadona e 2 $\mathrm{mcg} / \mathrm{kg}$ de Dexmedetomidina. Após o relaxamento do paciente, esse foi colocado no acesso venoso, onde foram administradas as drogas de indução anestésica, sendo $1 \mathrm{mg} / \mathrm{kg}$ de Ketamina e $3 \mathrm{mg} / \mathrm{kg}$ de Propofol. O paciente foi direcionado para o centro cirúrgico, realizou-se a intubação e a administração de drogas para a infusão contínua, sendo $5 \mathrm{mcg} / \mathrm{kg} / \mathrm{min}$ de Fentanil e $0,6 \mathrm{mg} / \mathrm{kg} / \mathrm{hora}$ de Ketamina.

Foi realizada a antissepsia do animal com clorexidine degermante e alcoólico, respeitando as áreas oftamológicas e mucosa. Iniciou-se o processo cirúrgico pela ressecção do palato mole alongado. A rima da glote deve ser usada como referência, uma vez que, a margem caudal do palato mole deve estar em contato com a ponta da epiglote e quando pressionada, contate o teto da nasofaringe dorsalmente. A medida é importante, pois se a ressecção for pequena, não resolve a síndrome braquicefálica. E se a ressecção for grande, pode causar rinite, sinusite e regurgitação nasal. (Fossum, 2014).

Utilizou - se uma atadura no maxilar do animal para abertura da cavidade oral. Três pinças Allis foram utilizadas para expor o palato mole (Imagem 2). Neste caso, foi usado o bisturi ultrassônico.

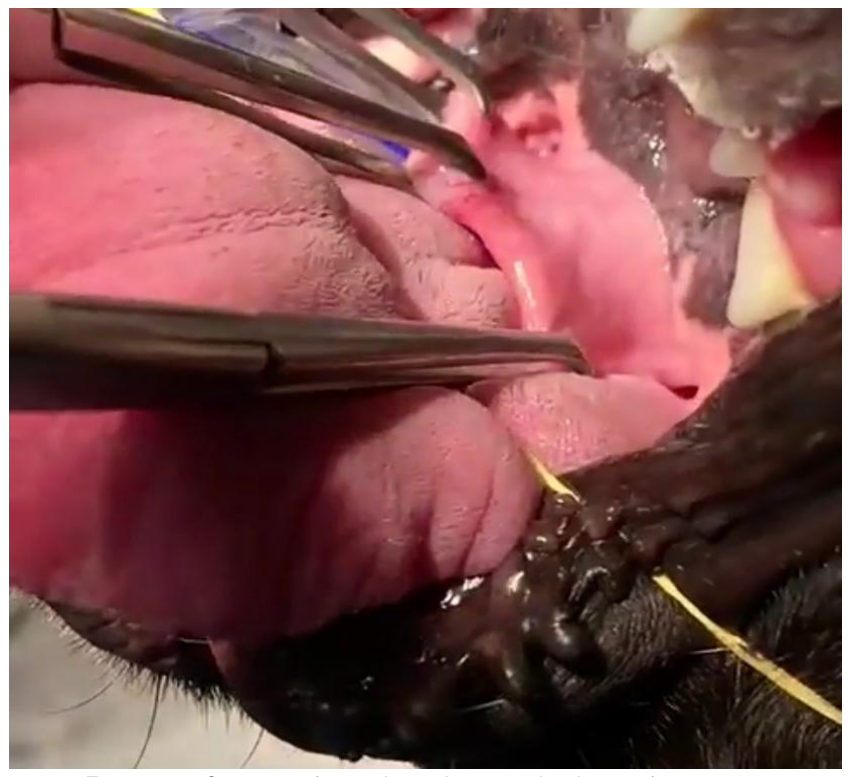

Imagem 2. Exposição do palato mole do paciente.

O bisturi ultrassônico possui uma onda de alta frequência, que quando transformada em energia mecânica, promove a secção dos vasos sanguíneos e de tecidos fibroadiposo e muscular. Esse recurso permite que se empregue menor tempo cirúrgico, além disso, o paciente tem menos perda sanguínea e formação de edema. Após a estafilectomia, o animal foi reposicionado sobre a mesa cirúrgica.

Durante a rinoplastia, a técnica utilizada foi a ressecção das bordas verticais segundo Fossum (2014). As margens das narinas foram presas e expostas com pinça Allis, realizando uma incisão em $\mathrm{V}$ ao redor da pinça com lâmina de bisturi número 11. A primeira incisão é medial, já a segunda lateral. A hemorragia foi controlada com pressão digital com gaze estéril. Após isso, foram alinhadas a margem ventral das narinas e a região muco cutânea, e realizadas 4 suturas simples contínuas com fio monofilamentar absorvível (Imagem 3).

O animal foi extubado e acordou sem complicações. Não foi realizada a correção cirúrgica da eversão sacos laríngeos, uma vez que, esses podem voltar à normalidade após a estafilectomia e rinoplastia. Essa decisão é particular de cada profissional, visto que alguns cirurgiões optam por realizar a correção de forma cirúrgica, mas outros não recomendam o procedimento (Poncet et al., 2006).

Foi receitado para o paciente em seu pós-cirúrgico, Rutina $30 \mathrm{mg} / \mathrm{kg} 24 / 24 \mathrm{~h}$, prednisolona $1 \mathrm{mg} / \mathrm{kg}$ $24 / 24 \mathrm{~h}$ durante cinco dias, $0,5 \mathrm{mg} / \mathrm{kg} 24 / 24 \mathrm{~h}$ durante três dias e $0,25 \mathrm{mg} / \mathrm{kg} 24 / 24 \mathrm{~h}$ durante dois dias, 


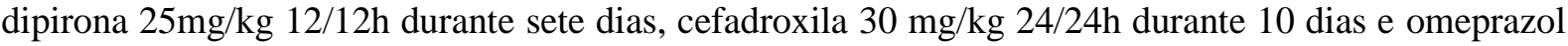
$0,5 \mathrm{mg} / \mathrm{kg} 12 / 12 \mathrm{~h}$ durante 10 dias.

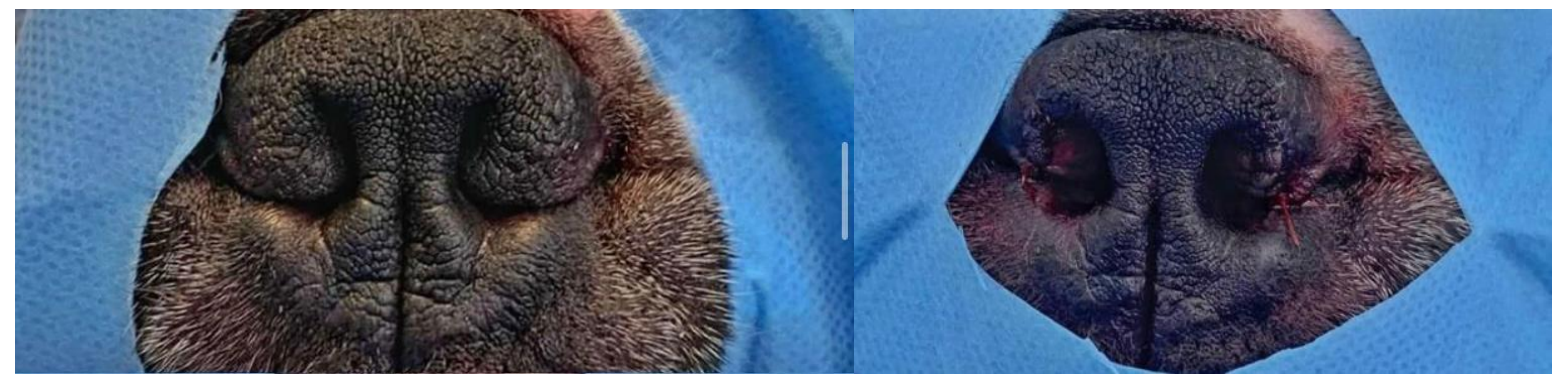

Imagem 3. Imagem anterior, narinas antes da rinoplastia e imagem posterior, narinas após a rinoplastia com uso da técnica de ressecção da borda vertical.

O paciente não precisou de internação pós-cirúrgica, pois com o uso do ultrassônico, o risco de edema e sangramento é muito baixo, permitindo que o paciente respire e se recupere de forma segura em casa, seguindo as medicações indicadas. $\mathrm{O}$ animal também já pode se alimentar normalmente após a procedimento, inicialmente dando preferência a alimentos em baixa temperatura.

A correção cirúrgica é a mais indicada, pois todos outros métodos são paliativos e não eficazes a longo prazo. Com a cirurgia, o animal tem maior qualidade de vida, além de longevidade, já que a síndrome braquicefálica se caracteriza por ser progressiva. As técnicas cirúrgicas também não são invasivas, por isso, a recuperação do animal é simples, sem grandes complicações (ㅍranco et al., 2015)

Além disso, o uso do bisturi ultrassônico facilita de maneira relevante a recuperação do paciente, que permaneceu sem formação de edema em seu pós-cirúrgico. O paciente se alimentou com alimentos pastosos no primeiro dia após a cirurgia, e no segundo dia, alimentou-se com ração seca. Dessa forma, esse tipo de bisturi facilita a recuperação tanto da região operada como do animal em si, que pode continuar sua rotina normalmente sem dor, estresse ou inapetência.

O paciente melhorou consideravelmente da síndrome braquicefálica, tornou-se tolerante ao exercício, também não apresentava mais ronco nem dormindo e nem acordado. $\mathrm{O}$ animal conseguia se alimentar normalmente, pois já não demonstrava disfagia ou regurgitação. Crises de espirro reverso também foram cessadas. A melhora do animal é imediata, visto que, ao acordar do procedimento, já consegue respirar sem dificuldade.

\section{Conclusão}

O prognóstico do animal é favorável após os procedimentos cirúrgicos, pois a estafilectomia e rinoplastia cessam significativamente os sinais clínicos da síndrome braquicefálica, logo após a cirurgia. É essencial o diagnóstico precoce para evitar manifestações secundárias e sequelas.

\section{Referências bibliográficas}

Fossum, T. W. (2014). Cirurgia de pequenos animais (4th ed., Vol. 1). Elsevier Brasil.

Franco, M. F., Dantas, W. M. F., Carvalho, T. B., \& Bergo, L. (2015). Prolongamento de palato moleEstafilectomia: Relato de caso. PUBVET, 9(6), 271-273.

Jericó, M. M., Kogika, M. M., \& Andrade Neto, J. P. (2015). Tratado de medicina interna de cães e gatos. Guanabara Koogan.

Lameu, G. R., Silva, P. Í. B., Menezes, A. D. R., Alves, C. C., Soares, M. A., Bilhalva, M. A., Evaristo, T. A., Pellegrin, T. G., Vasconcellos, A. L., \& Costa, P. P. C. (2020). Síndrome braquicefálica em cães: Revisão. PUBVET, 14(10), 1-7.

Lopes, B. G. P., \& Vasconcelos, T. C. (2020). Conhecimento de tutores sobre a síndrome aérea dos cães braquicefálicos. PUBVET, 15(6), 181.

Poncet, C. M., Dupre, G. P., Freiche, V. G., \& Bouvy, B. M. (2006). Long-term results of upper respiratory syndrome surgery and gastrointestinal tract medical treatment in 51 brachycephalic dogs. Journal of Small Animal Practice, 47(3), 137-142. 
Reinstein, R. S., Vogel, L. W., Degregori, E. B., Cassanego, G. R., Pozzobon, F. M., Ferreira, P. I., \& Müller, D. C. M. (2020). Reconstrução dermo-facial de canino com avulsão de lábio superior bilateral: Relato de caso. PUBVET, 15(5), 1-6. https://doi.org/10.31533/pubvet.v15n05a801.1-5.

Slatter, D. H. (2007). Manual de cirurgia de pequenos animais (Vol. 2). Manole São Paulo.

Histórico do artigo:

Recebido: 2 de julho de 2021

Aprovado: 24 de agosto de 2021

\begin{abstract}
Licenciamento: Este artigo é publicado na modalidade Acesso Aberto sob a licença Creative Commons Atribuição 4.0 (CC-BY 4.0), a qual permite uso irrestrito, distribuição, reprodução em qualquer meio, desde que o autor e a fonte sejam devidamente credita
\end{abstract}

Anexo 1. Laudo do exame hematológico (bioquímico)

Alanina aminotransferase (TGP / ALT) Canino
Data de Coleta............ 08/01/2021 13:05
Material biológico: Soro
Método: Enzimático/ automatizado
Resultado.................63 U/L 63

\section{Creatinina Canino}

Data de Coleta............... 08/01/2021 13:05
Material biológico: Soro
Método: Enzimático
Resultado.................... $\mathbf{0 , 9}$ mg/dL

\section{Fosfatase Alcalina Canino}

Data de Coleta 08/01/2021 13:05

Material biológico: Soro

Método: Enzimático 45

U/L

Valores Referenciais:

Resultado

20 a $156 \mathrm{U} / \mathrm{L}$

\section{Uréia Canino}

Data de Coleta 08/01/2021 13:05

Material biológico: Soro

Método: Enzimático / automatizado

Valores Referenciais:

Resultado.

23

$\mathrm{mg} / \mathrm{dL}$

10 a $60 \mathrm{mg} / \mathrm{dL}$

Observação

Exames confirmados em duplicata.

Soro discretamente hemolisado

Anexo 2. Laudo do exame hematológico (pesquisa de microfilária)

\section{Pesquisa de Microfilária}

Data de Coleta. $08 / 01 / 2021$

Material biológico: Sangue Total em EDTA

Método: Knott modificado

Resultado Não foram encontradas microfilárias na amostra enviada. 
Anexo 3 Laudo do exame hematológico (hemograma)

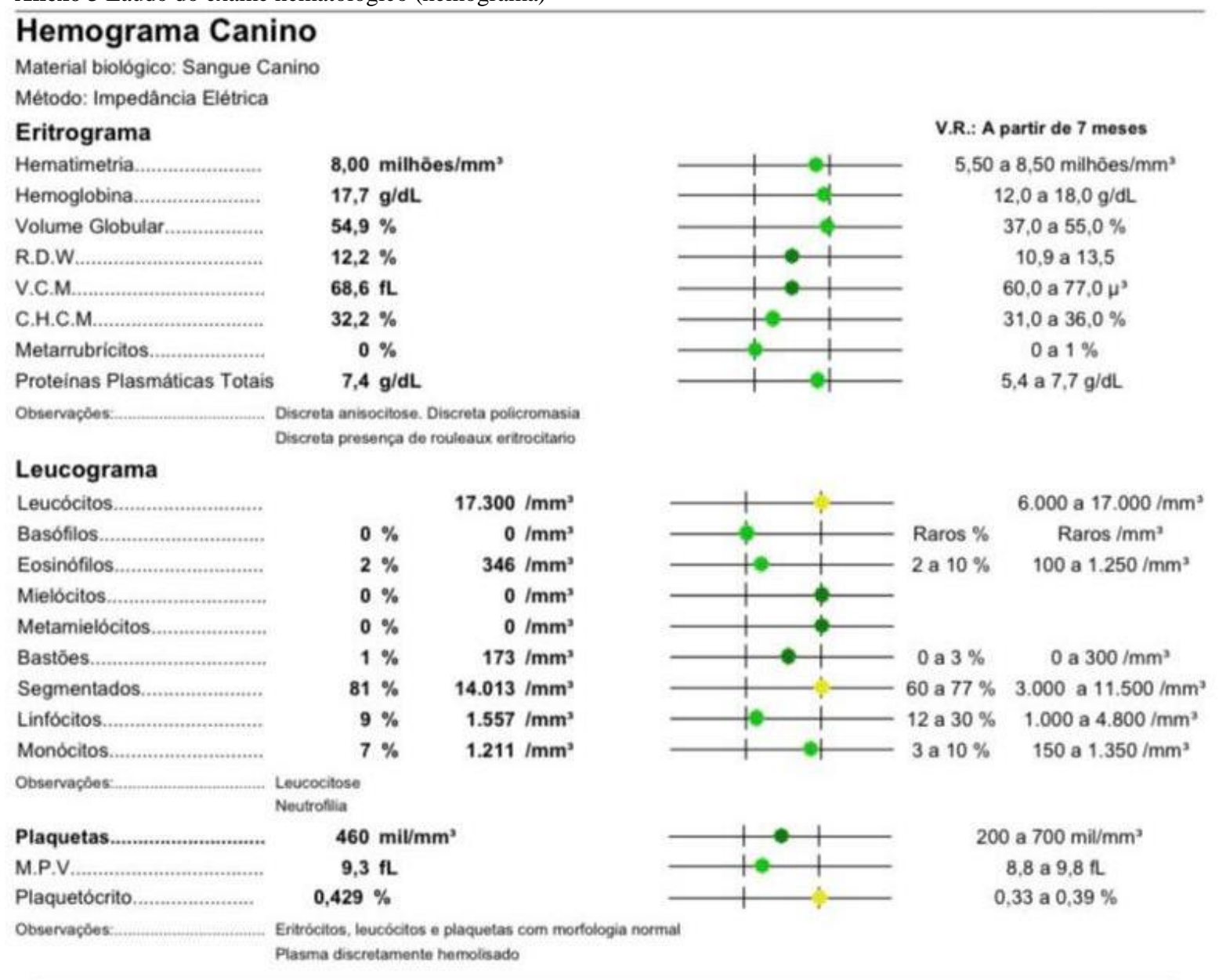

Anexo 4. Laudo do eletrocardiograma do paciente

\section{Parâmetros Observados}

Eixo QRS: $48.39^{\circ}$

Eixo P: $27.96^{\circ}$

Segmento ST: $80 \mathrm{~ms}$

Intervalo PR: $94 \mathrm{~ms}$

Duração de P: $40 \mathrm{~ms}$
Duração de T: $78 \mathrm{~ms}$

Duração de QRS: $58 \mathrm{~ms}$

Desnivel de ST: $-0.06 \mathrm{mV}$

Amplitude de P: $0.08 \mathrm{mV}$

Amplitude de R: $1.31 \mathrm{mV}$
Amplitude de S: $-0.09 \mathrm{mV}$

Intervalo QT: $216 \mathrm{~ms}$

Amplitude de T: $-0.43 \mathrm{mV}$

FC Média: 91 bpm

\section{Comentários}

Paciente agitado durante o exame

Ritmo irregularmente irregular

Onda P: Sem alteração

Complexo QRS: Sem alteração

Onda T: Sem alteração

Intervalo PR: Sem alteração

Desnivel ST: Sem alteração

\section{Conclusões}

Arritmia sinusal com frequência cardiaca mínima de 64 bpm e máxima de 151 bpm. 